\title{
The spectral excess theorem for distance-regular graphs: a global (over)view
}

\author{
Edwin R. van Dam \\ Tilburg University, Dept. Econometrics \& O.R. \\ PO Box 90153, 5000 LE Tilburg, The Netherlands \\ Edwin.vanDam@uvt.nl \\ Submitted: April 24, 2008; Accepted: Oct 3, 2008; Published: Oct 13, 2008 \\ Mathematics Subject Classification: 05E30, 05B20
}

Keywords: distance-regular graphs, eigenvalues of graphs, spectral excess theorem

\begin{abstract}
Distance-regularity of a graph is in general not determined by the spectrum of the graph. The spectral excess theorem states that a connected regular graph is distance-regular if for every vertex, the number of vertices at extremal distance (the excess) equals some given expression in terms of the spectrum of the graph. This result was proved by Fiol and Garriga [From local adjacency polynomials to locally pseudo-distance-regular graphs, J. Combinatorial Th. B 71 (1997), 162-183] using a local approach. This approach has the advantage that more general results can be proven, but the disadvantage that it is quite technical. The aim of the current paper is to give a less technical proof by taking a global approach.
\end{abstract}

\section{Introduction}

It is known that distance-regularity of a graph is in general not determined by the spectrum of the graph, cf. [7] and [11] for recent results on spectral characterizations of distance-regular graphs.

By the spectral excess theorem we mean the remarkable result by Fiol and Garriga [13] that a connected regular graph with $d+1$ distinct eigenvalues is distance-regular if for every vertex, the number of vertices at distance $d$ from that vertex (the excess) equals a given expression in terms of the spectrum. So besides the spectrum, a simple combinatorial property suffices for a graph to be distance-regular.

The first result in this direction was obtained by Cvetković [2] and by Laskar [18], who showed that for a Hamming graph with diameter three (and consequently a Doob graph with diameter three), distance-regularity is determined by the spectrum and having the correct number of vertices at distance two (or, equivalently, three) from each vertex. 
This result was generalized to all distance-regular graphs with diameter three by Haemers [16], after which he and the author [5] showed the spectral excess theorem for graphs with four distinct eigenvalues. The difference between the two results is that in the latter it is not assumed that the graph has the spectrum of a distance-regular graph.

At the same time, Fiol et al. [14] showed that a graph with $d+1$ distinct eigenvalues is distance-regular if each vertex has at least one vertex at distance $d$ and its distance- $d$ adjacency matrix $A_{d}$ is a polynomial of degree $d$ in the adjacency matrix $A$. This result is halfway towards the spectral excess theorem, which was then proved by Fiol and Garriga in [13]. A slight improvement of it, which is proved here as Theorem 1, was later proved in $[11]$.

Similar spectral characterization results on three-class association schemes were obtained by the author [4], which were generalized again by Fiol [10]. Fiol [9] also obtained a more specific result for antipodal distance-regular graphs.

The usefulness of the spectral excess theorem is for example demonstrated in the recent discovery of a new family of distance-regular graphs with the same parameters of particular Grassmann graphs, cf. [8]. It is shown there that the new graphs have the same spectrum as the Grassmann graphs, and then counting the number of vertices at extremal distance proves the distance-regularity.

The goal of this paper is to give an elementary and global proof of the spectral excess theorem. The original proof by Fiol et al. [13, 14] has a local approach and is quite technical because of that. It turns out that restricting to regular graphs allows some shortcuts and makes a global approach towards a proof possible. We want to stress however that the essential steps in the given proof are by Fiol et al. The intention here is to give a streamlined, accessible, and self-contained proof as far as possible. We also remark that by their local approach, Fiol et al. manage to prove more results.

We assume the reader has basic knowledge of linear algebra and spectra of graphs. For background on the latter we refer the reader to the book by Cvetković, Doob, and Sachs [3]. For distance-regular graphs, see the book by Brouwer, Cohen, and Neumaier [1]. For several topics on algebraic combinatorics, such as distance-regular graphs and orthogonal polynomials, the book by Godsil [15] will be useful. Finally, we refer the reader who has become interested in the topic to recent surveys by Fiol $[11,12]$.

\section{Basic preliminaries}

We only consider simple undirected graphs, i.e., there are no loops or multiple edges.

\subsection{Distance-regular graphs}

A connected graph $\Gamma$ is called distance-regular, with diameter $d$, if there are constants $a_{i}, b_{i}, c_{i}, i=0,1, \ldots, d$ such that for any $i=0,1, \ldots, d$, and any two vertices $x$ and $y$ at distance $i$, among the neighbours of $y$, there are $c_{i}$ at distance $i-1$ from $x, a_{i}$ at distance $i$, and $b_{i}$ at distance $i+1$. A distance-regular graph is regular with valency $k:=b_{0}$. 
We denote the number of vertices of $\Gamma$ by $n$. The mentioned constants are called the intersection parameters.

Let us denote by $\Gamma_{i}$ the distance- $i$ graph of $\Gamma$, i.e., $x$ and $y$ are adjacent in $\Gamma_{i}$ if and only if they are at distance $i$ in $\Gamma$. Also, let $\Gamma_{i}(x)$ be the set of vertices at distance $i$ from $x$, and let $\Gamma(x)=\Gamma_{1}(x)$ be the set of neighbours of $x$.

In order to translate the definition of distance-regularity into a more algebraic one, we define the distance- $i$ adjacency matrix $A_{i}$ as the adjacency matrix of $\Gamma_{i}$. Thus $\left(A_{i}\right)_{x y}$ equals one if $x$ and $y$ are at distance $i$ in $\Gamma$, and zero otherwise. We denote by $A=A_{1}$ the usual adjacency matrix. Now the above definition of distance-regular graphs is equivalent to the equations

$$
A A_{i}=c_{i+1} A_{i+1}+a_{i} A_{i}+b_{i-1} A_{i-1} \text { for } i=0,1, \ldots, d .
$$

The so-called distance polynomials of a distance-regular graph form a family of orthogonal polynomials. These distance polynomials $p_{i}, i=0,1, \ldots, d$ are defined by $p_{0}(\theta)=1$, and the three-term recurrence relation $\theta p_{i}=c_{i+1} p_{i+1}+a_{i} p_{i}+b_{i-1} p_{i-1}$ for $i=0,1, \ldots, d$ (to be precise we have to take $b_{-1} p_{-1}=c_{d+1} p_{d+1}=0$ ). This is clearly motivated by the above equations for the distance- $i$ matrices, which now satisfy $A_{i}=p_{i}(A), i=0,1, \ldots, d$.

Now let us define an inner product on the vector space of polynomials of degree at most $d$ by $\langle p, q\rangle=\frac{1}{n} \operatorname{tr}(p(A) q(A))$. Then the distance polynomials satisfy $\left\langle p_{i}, p_{j}\right\rangle=$ $\frac{1}{n} \operatorname{tr}\left(p_{i}(A) p_{j}(A)\right)=\frac{1}{n} \operatorname{tr}\left(A_{i} A_{j}\right)=0$ if $i \neq j$, i.e., they are a set of orthogonal polynomials.

In Section 4.1, we shall define orthogonal polynomials for any regular graph, using the spectrum of the graph, in an attempt to generalize the distance polynomials. These

othogonal polynomials will form the key component of the spectral excess theorem.

\subsection{Spectrum of a graph}

Let $\Gamma$ be a connected regular graph with adjacency matrix $A$ and spectrum

$$
\Sigma=\left\{\lambda_{0}^{m_{0}}, \lambda_{1}^{m_{1}}, \ldots, \lambda_{d}^{m_{d}}\right\}
$$

where the superscripts $m_{i}$ denote the multiplicities of the distinct eigenvalues $\lambda_{i}, i=$ $0,1, \ldots, d$, and where $k:=\lambda_{0}$ is the valency (and largest eigenvalue), with multiplicity $m_{0}=1$.

By $E_{i}$ we denote the matrix representing the projection onto the eigenspace $\operatorname{ker}(A-$ $\left.\lambda_{i} I\right)$, i.e., $E_{i}=U_{i} U_{i}^{\top}$, where $U_{i}$ has as columns an orthonormal basis of the eigenspace. It now follows that $E_{i} E_{j}=O$ for $i \neq j, E_{i}^{2}=E_{i}, \operatorname{tr}\left(E_{i}\right)=m_{i}$, and $p(A)=\sum_{i=0}^{d} p\left(\lambda_{i}\right) E_{i}$ for any polynomial $p$.

It is known that the intersection parameters of a distance-regular graph determine its spectrum. In particular, if its diameter is $d$, then it has $d+1$ distinct eigenvalues. It also works the other way around: the spectrum of a distance-regular graph determines its intersection parameters. However, it is in general not true that the spectrum of a graph determines that it is distance-regular. In the following section we give an example where it does not. We shall use this example as an illustration of the spectral excess theorem. 


\section{The four-dimensional cube and the Hoffman graph}

The four-dimensional cube, or Hamming graph $H(4,2)$, is a distance-regular graph with spectrum $\Sigma=\left\{4^{1}, 2^{4}, 0^{6},-2^{4},-4^{1}\right\}$. Hoffman [17] determined all graphs with this spectrum, thus finding another graph, that is now called the Hoffman graph. As an introduction to the spectral excess theorem, we will have a closer look at the properties of graphs with spectrum $\Sigma$.

Let $\Gamma$ be a graph with spectrum $\Sigma$. From basic theory of graph spectra (cf. [6]), we find that $\Gamma$ is a connected, bipartite, regular graph with valency 4, on 16 vertices, with diameter at most 4. Hoffman [17] introduced the now-called Hoffman-polynomial to determine that the adjacency matrix $A$ of $\Gamma$ satisfies the equation $(A-2 I) A(A+2 I)(A+4 I)=24 J$, which can also be written as $A^{4}+4 A^{3}-4 A^{2}-16 A=24 J$. Let us now think about the constants $a_{i}, b_{i}, c_{i}$ that we would like to have in order for $\Gamma$ to be distance-regular. Trivially $b_{0}=k=4$ and $c_{1}=1$ are well-defined (that is, they satisfy the properties as in the definition of a distance-regular graph). Because $\Gamma$ is bipartite, $a_{i}=0$ is well-defined for each relevant $i$. This implies that also $b_{1}=k-a_{1}-c_{1}=3$ and $c_{4}=k-a_{4}=4$ are well-defined. Moreover, the bipartiteness and the fact that the diameter is at most 4 together imply that each vertex has $4\left(=\frac{16}{2}-4\right)$ vertices at distance 3 . Now we fix a vertex $x$, and let $c_{2}(x, y)$ be the number of common neighbours of $x$ and $y$, for $y$ at distance 2 from $x$. Between the neighbours of $x$ and the vertices at distance 2 from $x$ there are $k b_{1}=12$ edges, so it follows that

$$
\sum_{y \in \Gamma_{2}(x)} c_{2}(x, y)=12 .
$$

Because $\left(A^{\ell}\right)_{x x}$ counts the number of so-called closed walks from $x$ to itself of length $\ell$, it follows that $\left(A^{\ell}\right)_{x x}=0$ for all odd $\ell$ (in fact, this is how bipartiteness from the spectrum can be proven, as $\operatorname{tr}\left(A^{\ell}\right)=0$ for odd $\ell$ implies that there are no odd cycles). Specifying the Hoffman-polynomial for the diagonal position of $x$ gives that $\left(A^{4}\right)_{x x}=4\left(A^{2}\right)_{x x}+24=40$. Elementary counting of the closed walks of length 4 from $x$ to itself now gives that

$$
\sum_{y \in \Gamma_{2}(x)} c_{2}(x, y)^{2}=24 .
$$

If $k_{i}(x)$ denotes the number of vertices at distance $i$ from $x$, then it follows from Cauchy's inequality that

$$
24=\sum_{y \in \Gamma_{2}(x)} c_{2}(x, y)^{2} \geq \frac{1}{k_{2}(x)}\left[\sum_{y \in \Gamma_{2}(x)} c_{2}(x, y)\right]^{2}=\frac{144}{k_{2}(x)}
$$

hence $k_{2}(x) \geq 6$, and more importantly, if equality holds, then $c_{2}(x, y)$ is the same for all $y \in \Gamma_{2}(x)$. The conclusion is that if $k_{2}(x)=6$ for all $x$, or equivalently, if $k_{4}(x)=1$ for all $x$, then $c_{2}=2$ is well-defined, and then it follows easily that $\Gamma$ is distance-regular. This conclusion is the spectral excess theorem for this case. 
By further working out the cases, Hoffman determined that besides the distanceregular 4-dimensional cube, there is one other - non-distance-regular - graph with spectrum $\Sigma$. An easy construction of this Hoffman graph is obtained by "switching" the 12 edges and 12 non-edges between the neighbours and vertices at distance 2 of a fixed vertex, cf. [7].

\section{More ingredients}

Let us now prepare some more necessary ingredients for the spectral excess theorem.

\subsection{Inner product, orthogonal polynomials, three-term recur- rence}

From the spectrum $\Sigma=\left\{\lambda_{0}^{m_{0}}, \lambda_{1}^{m_{1}}, \ldots, \lambda_{d}^{m_{d}}\right\}$ we define an inner product on the vector space of polynomials of degree at most $d$ by

$$
\langle p, q\rangle=\frac{1}{n} \sum_{i=0}^{d} m_{i} p\left(\lambda_{i}\right) q\left(\lambda_{i}\right) .
$$

This is indeed a well-defined inner product (a symmetric bilinear product for which $\langle p, p\rangle \geq 0$ with equality if and only if $p=0$ ). Moreover, for any graph with adjacency matrix $A$ and spectrum $\Sigma$, we have that

$$
\langle p, q\rangle=\frac{1}{n} \operatorname{tr}(p(A) q(A))
$$

which agrees with the definition for distance-regular graphs in Section 2.1.

With respect to this inner product, there is a unique system of orthogonal polynomials $p_{i}, i=0,1, \ldots, d$, where $p_{i}$ has degree $i$ and

$$
\left\langle p_{i}, p_{i}\right\rangle=p_{i}\left(\lambda_{0}\right) \text { for } i=0,1, \ldots, d .
$$

This system can be obtained by applying the Gram-Schmidt procedure to the basis of monomials $1, \theta, \ldots, \theta^{d}$ and normalizing. The latter can be done because $p_{i}\left(\lambda_{0}\right)>0$ (this is well-known: if $p_{i}$ changes sign at values $\theta_{j}, j=1,2, \ldots, h$ in the interval $\left(\lambda_{d}, \lambda_{0}\right)$, and $q(\theta)=\prod_{j=1}^{h}\left(\theta-\theta_{j}\right)$, then $p_{i}(\theta) q(\theta) \geq 0$ for $\lambda_{d} \leq \theta \leq \lambda_{0}$ and even better $\left\langle p_{i}, q\right\rangle>0$, which implies that $h=i$, and that all roots of $p_{i}$ are distinct and in the interval $\left(\lambda_{d}, \lambda_{0}\right)$; moreover, the leading term of $p_{i}$ is positive).

As with the distance-regular graphs, we can obtain a three-term recurrence relation for the orthogonal polynomials. This is well-known from the theory of orthogonal polynomials (cf. [15]), but also easily explained as follows. The defined inner product on polynomials satisfies an extra useful property, namely that $\langle\theta p, q\rangle=\langle p, \theta q\rangle$. This can be used when 
we consider the polynomial $\theta p_{i}$ which is of degree $i+1$, and hence can be expressed as $\theta p_{i}=\sum_{j=0}^{i+1} \alpha_{i j} p_{j}$ for certain $\alpha_{i j}$. These satisfy $\alpha_{i j}\left\langle p_{j}, p_{j}\right\rangle=\left\langle\theta p_{i}, p_{j}\right\rangle=\left\langle p_{i}, \theta p_{j}\right\rangle$. The right hand side is zero for $j<i-1$, and hence so is $\alpha_{i j}$. Thus only three terms remain in the expression of $\theta p_{i}$. After renaming the constants, we obtain the (familiar) three-term recurrence relation

$$
\theta p_{i}=c_{i+1} p_{i+1}+a_{i} p_{i}+b_{i-1} p_{i-1} \text { for } i=0,1, \ldots, d .
$$

In the above, we have to be a bit more careful in the cases $i=0$ and $i=d$. In the first case, the above arguments are valid if we take $b_{-1} p_{-1}=0$. In the latter case, the polynomial $\theta p_{d}$ is however not a polynomial of degree at most $d$, and hence it seems that we cannot take inner products with this polynomial. However, we can reduce the polynomial to a polynomial of degree at most $d$ by subtracting an appropriate multiple of the minimal polynomial $m(\theta)=\prod_{i=0}^{d}\left(\theta-\lambda_{i}\right)$. This does not change the inner product, and it also does not change the application of the polynomial to the adjacency matrix $A$ with spectrum $\Sigma$, because $m(A)=O$. We thus may take $c_{d+1}=0$. (In fact, we are working with the set of all polynomials modulo the minimal polynomial.)

It furthermore follows that $c_{i+1}=\frac{\left\langle\theta p_{i}, p_{i+1}\right\rangle}{\left\langle p_{i+1}, p_{i+1}\right\rangle} \neq 0$ for $i<d$ and $b_{i-1}=\frac{\left\langle\theta p_{i}, p_{i-1}\right\rangle}{\left\langle p_{i-1}, p_{i-1}\right\rangle}=$ $\frac{\left\langle p_{i}, \theta p_{i-1}\right\rangle}{\left\langle p_{i-1}, p_{i-1}\right\rangle} \neq 0$ for $i>0$.

We note that in general, there seems to be no easy combinatorial interpretation of the obtained constants $a_{i}, b_{i}, c_{i}$, except for distance-regular graphs, of course.

\subsection{Optimality of the polynomials, and the Hoffman-polynomial}

In the case of distance-regular graphs, we have that $\sum_{i=0}^{d} p_{i}(A)=J$, the all-one matrix. We shall show that this holds for any (connected) regular graph. This follows from an optimality property of the partial sums of the polynomials $p_{i}$. Let these partial sums be defined by $q_{i}=\sum_{j=0}^{i} p_{j}$. We thus claim that

$$
q_{d}(A)=J
$$

or in other words, $q_{d}$ is the well-known Hoffman-polynomial [17].

To prove the claim, we first show that $q_{i}$ is the (unique) polynomial $p$ of degree $i$ that maximizes $p(k)$ subject to the constraint that $\langle p, p\rangle=\left\langle q_{i}, q_{i}\right\rangle$. To show this property, write a polynomial $p$ of degree $i$ as $p=\sum_{j=0}^{i} \alpha_{j} p_{j}$ for certain $\alpha_{j}$ (for fixed $i$ ). Then the problem reduces to maximizing $p(k)=\sum_{j=0}^{i} \alpha_{j} p_{j}(k)$ subject to $\sum_{j=0}^{i} \alpha_{j}^{2} p_{j}(k)=\left\langle q_{i}, q_{i}\right\rangle$. Now Cauchy's inequality implies that

$$
p(k)^{2}=\left[\sum_{j=0}^{i} \alpha_{j} p_{j}(k)\right]^{2} \leq\left[\sum_{j=0}^{i} \alpha_{j}^{2} p_{j}(k)\right]\left[\sum_{j=0}^{i} p_{j}(k)\right]=q_{i}(k)^{2},
$$

with equality if and only if all $\alpha_{j}$ are equal. The constraint and the fact that $p_{j}(k)>0$ for all $j$ guarantees that $q_{i}$ is the optimal $p$. 
On the other hand, since $\langle p, p\rangle=\frac{1}{n} p(k)^{2}+\frac{1}{n} \sum_{j=1}^{d} m_{j} p\left(\lambda_{j}\right)^{2}$, the objective of the optimization problem is clearly equivalent to minimizing $\sum_{j=1}^{d} m_{j} p\left(\lambda_{j}\right)^{2}$. For $i=d$, there is a trivial solution for this: take the polynomial that is zero on $\lambda_{j}$ for all $j=1,2, \ldots, d$. Hence we may conclude that $q_{d}\left(\lambda_{j}\right)=0$ for $j=1,2, \ldots, d$, and from the constraint it further follows that $q_{d}(k)=n$.

Now $q_{d}(A)=\sum_{i=0}^{d} q_{d}\left(\lambda_{i}\right) E_{i}=q_{d}(k) E_{0}=J$.

\subsection{Moving forward to go back: the conjugate polynomials}

The earlier mentioned reduction of polynomials modulo the minimal polynomial will turn out to be crucial in the proof of the spectral excess theorem. Loosely speaking, it will be used to move back from the vertices at extremal distance $d$ to the vertices at any other fixed distance $d-i$ by moving "forward" $i$ steps. For now, this will be presented in the form of the existence of the so-called conjugate polynomials $\bar{p}_{i}$ of degree $i$, for $i=0,1, \ldots, d$, with the property that

$$
p_{d-i}(A)=\bar{p}_{i}(A) p_{d}(A) \text { for } i=0,1, \ldots, d \text {. }
$$

We shall prove this by induction. For $i=0$, the existence is trivial. From the three-term recurrence it follows that $p_{d-i-1}(A)=\frac{1}{b_{d-i-1}}\left[\left(A-a_{d-i} I\right) p_{d-i}(A)-c_{d-i+1} p_{d-i+1}(A)\right]$, which provides the induction steps (for $i=0$ it gives the step from 0 to 1 because $c_{d+1}=0$ ).

\section{The spectral excess theorem}

We are ready now for the spectral excess theorem. We shall state and prove it in a bit stronger form than earlier stated.

Theorem 1. Let $\Gamma$ be a connected k-regular graph on $n$ vertices with spectrum $\Sigma$ with corresponding orthogonal polynomials $p_{i}, i=0,1, \ldots, d$. If $k_{d}(x)$ is the number of vertices at distance $d$ from $x$, then

$$
\frac{n}{\sum_{x} \frac{1}{n-k_{d}(x)}} \geq n-p_{d}(k)
$$

with equality if and only if $\Gamma$ is distance-regular.

So, instead of requiring that $k_{d}(x)=p_{d}(k)$ for all $x$, we require that the harmonic mean of the $n-k_{d}(x)$ equals $n-p_{d}(k)$. We shall prove the theorem in two steps:

Lemma 1. $\frac{n}{\sum_{x} \frac{1}{n-k_{d}(x)}} \geq n-p_{d}(k)$ with equality if and only if $A_{d}=p_{d}(A)$.

Proof. We have that

$$
q_{d-1}(k)=\left\langle q_{d-1}, q_{d-1}\right\rangle=\frac{1}{n} \operatorname{tr}\left(q_{d-1}(A)^{2}\right)=\frac{1}{n} \sum_{x}\left(q_{d-1}(A)^{2}\right)_{x x}=\frac{1}{n} \sum_{x} \sum_{y \notin \Gamma_{d}(x)}\left(q_{d-1}(A)_{x y}\right)^{2}
$$




$$
\geq \frac{1}{n} \sum_{x} \frac{1}{n-k_{d}(x)}\left[\sum_{y \notin \Gamma_{d}(x)} q_{d-1}(A)_{x y}\right]^{2}=\frac{1}{n} \sum_{x} \frac{1}{n-k_{d}(x)}\left[q_{d-1}(k)\right]^{2} .
$$

The stated inequality now follows from the fact that $n-p_{d}(k)=q_{d-1}(k)$. If equality holds, then it follows from the above that for each $x$ the values of $q_{d-1}(A)_{x y}$ are the same for all $y \notin \Gamma_{d}(x)$. This implies that $q_{d-1}(A)_{x y}$ is the same for each pair of vertices $x$ and $y$ at distance less than $d$. Since $q_{d-1}(A)$ has constant row sums $\left(q_{d-1}(k)\right)$, it follows that each vertex has the same number of vertices at distance less than $d$. From the equality it follows that this number must be $q_{d-1}(k)$, and hence $q_{d-1}(A)_{x y}=1$ if $x$ and $y$ are at distance less than $d$. Thus $q_{d-1}(A)=J-A_{d}$, and hence $A_{d}=p_{d}(A)$. Conversely, if $A_{d}=p_{d}(A)$ then the row sums give that $k_{d}(x)=p_{d}(k)$ for every vertex $x$, and equality holds.

We then use the conjugate polynomials to prove the following.

Lemma 2. If $A_{d}=p_{d}(A)$, then $A_{i}=p_{i}(A)$ for all $i=0,1, \ldots, d$.

Proof. Because $p_{i}$ is a polynomial of degree $i$, it follows that if $x$ and $y$ are two vertices at distance larger than $i$, then $p_{i}(A)_{x y}=0$. Suppose now that $A_{d}=p_{d}(A)$. Then $p_{i}(A)=\bar{p}_{d-i}(A) A_{d}$. If the distance between $x$ and $y$ is smaller than $i$, then for all vertices $z$ at distance $d$ from $y$, we have that the distance between $z$ and $x$ is more than $d-i$ (by the triangle inequality), hence $\left(\bar{p}_{d-i}\right)_{x z}=0$. Thus

$$
\left(p_{i}(A)\right)_{x y}=\left(\bar{p}_{d-i}(A) A_{d}\right)_{x y}=\sum_{z}\left(\bar{p}_{d-i}(A)\right)_{x z}\left(A_{d}\right)_{z y}=0
$$

Because this holds for all $i=0,1, \ldots, d$ and because $\sum_{i=0}^{d} p_{i}(A)=q_{d}(A)=J$, it follows that $p_{i}(A)=A_{i}$ for all $i=0,1, \ldots, d$.

Now the proof of the spectral excess theorem is complete: if $A_{i}=p_{i}(A)$ for all $i$, then the three-term recurrence relation for the polynomials becomes the required recurrence for the adjacency matrices $A_{i}$, which proves the distance-regularity.

\section{An expression for the spectral excess}

The spectral excess $p_{d}(k)$ can be computed from the spectrum more directly as

$$
p_{d}(k)=\frac{n}{\pi_{0}^{2}}\left[\sum_{i=0}^{d} \frac{1}{m_{i} \pi_{i}^{2}}\right]^{-1}
$$

where $\pi_{i}=\prod_{j \neq i}\left|\lambda_{i}-\lambda_{j}\right|$ for $i=0,1, \ldots, d$. We can derive this expression by considering the polynomials $h_{i}=\prod_{j \neq 0, i}\left(x-\lambda_{j}\right)$, for $i=1,2, \ldots, d$. These polynomials have degree 
$d-1$, hence they are orthogonal to $p_{d}$. Thus,

$$
0=n\left\langle p_{d}, h_{i}\right\rangle=\sum_{j=0}^{d} m_{j} p_{d}\left(\lambda_{j}\right) h_{i}\left(\lambda_{j}\right)=p_{d}(k) h_{i}(k)+m_{i} p_{d}\left(\lambda_{i}\right) h_{i}\left(\lambda_{i}\right)
$$

which implies that $p_{d}\left(\lambda_{i}\right)=-\frac{p_{d}(k) h_{i}(k)}{m_{i} h_{i}\left(\lambda_{i}\right)}$ for $i=1,2, \ldots, d$. By substituting this into the equation $p_{d}(k)=\frac{1}{n} \sum_{i=0}^{d} m_{i} p_{d}\left(\lambda_{i}\right)^{2}$, and working out the details, the above expression follows.

\section{Open problem}

For so-called walk-regular graphs, the inequality in Lemma 1 can be improved to an inequality for each vertex. A graph is called walk-regular if for each $\ell$, the number of closed walks of length $\ell$ from a vertex $x$ to itself is the same for each $x$. In other words, if $A^{\ell}$ has constant diagonal for every $\ell$. If this is the case, then $q_{d-1}(A)^{2}$ also has constant diagonal, and after adjusting the proof of Lemma 1 , it follows that $k_{d}(x) \leq p_{d}(k)$ for every vertex $x$. It is however an open problem whether these inequalities hold for all regular graphs. Note that all regular graphs with at most four distinct eigenvalues are walk-regular, and so are the bipartite regular graphs with five eigenvalues, such as the four-dimensional cube and the Hoffman graph (where we indeed derived the inequalities).

Acknowledgements The author would like to thank Jack Koolen and his students at POSTECH for the warm hospitality and inspiration during a visit in February 2008.

\section{References}

[1] A.E. Brouwer, A.M. Cohen, and A. Neumaier. Distance-Regular Graphs, SpringerVerlag, Heidelberg, 1989.

[2] D.M. Cvetković, New characterizations of the cubic lattice graphs, Publ. Inst. Math. (Beograd) 10 (1970), 195-198.

[3] D.M. Cvetković, M. Doob, and H. Sachs, Spectra of Graphs, third edition, Johann Ambrosius Barth Verlag, 1995. (First edition: Deutscher Verlag der Wissenschaften, Berlin 1980; Academic Press, New York 1980.)

[4] E.R. van Dam, Bounds on special subsets in graphs, eigenvalues and association schemes, J. Algebraic Combinatorics 7 (1998), 321-332.

[5] E.R. van Dam and W.H. Haemers, A characterization of distance-regular graphs with diameter three, J. Algebraic Combinatorics 6 (1997), 299-303.

[6] E.R. van Dam and W.H. Haemers, Which graphs are determined by their spectrum?, Linear Algebra Appl. 373 (2003), 241-272. 
[7] E.R. van Dam, W.H. Haemers, J.H. Koolen, and E. Spence, Characterizing distanceregularity of graphs by the spectrum, J. Combinatorial Th. A 113 (2006), 1805-1820.

[8] E.R. van Dam and J.H. Koolen, A new family of distance-regular graphs with unbounded diameter, Inventiones Mathematicae 162 (2005), 189-193.

[9] M.A. Fiol, An eigenvalue characterization of antipodal distance-regular graphs, Electronic J. Combinatorics 4 (1997), R30.

[10] M.A. Fiol, Some applications of the proper and adjacency polynomials in the theory of graph spectra, Electronic J. Combinatorics 4 (1997), R21.

[11] M.A. Fiol, Algebraic characterizations of distance-regular graphs, Discrete Math. 246 (2002), 111-129.

[12] M.A. Fiol, Spectral bounds and distance-regularity, Linear Algebra Appl. 397 (2005), $17-33$.

[13] M.A. Fiol and E. Garriga, From local adjacency polynomials to locally pseudodistance-regular graphs, J. Combinatorial Th. B 71 (1997), 162-183.

[14] M.A. Fiol, E. Garriga, and J.L.A. Yebra, Locally pseudo-distance-regular graphs, $J$. Combinatorial Th. B 68 (1996), 179-205.

[15] C.D. Godsil, Algebraic Combinatorics, Chapman \& Hall, New York, 1993.

[16] W.H. Haemers, Distance-regularity and the spectrum of graphs, Linear Algebra Appl. 236 (1996), 265-278.

[17] A.J. Hoffman, On the polynomial of a graph, Amer. Math. Monthly 70 (1963), 30-36.

[18] R. Laskar, Eigenvalues of the adjacency matrix of the cubic lattice graph, Pacific J. Math. 29 (1969), 623-629. 